\title{
XVIII. Observations on the blood corpuscles, or red particles, of the mammiferous animals
}

\section{George Gulliver F.R.S.}

To cite this article: George Gulliver F.R.S. (1840) XVIII. Observations on the blood corpuscles, or red particles, of the mammiferous animals, Philosophical Magazine Series 3, 16:101, 105-115, DOI: $10.1080 / 14786444008649990$

To link to this article: http://dx.doi.org/10.1080/14786444008649990

册 Published online: 01 Jun 2009.

Submit your article to this journal $₫$

Џll Article views: 1

Q View related articles $₫$ 
XVIII. Observations on the Blood Corpuscles, or Red Particles, of the Mammiferous Animals. By George Gulliver, F.R.S., Assistant Surgeon to the Royal Regiment of Horse Guards. No. II.*

TN my former communication + I omitted to mention that 1 in many of the observations an achromatic object glass was used, of one tenth of an inch focal length, made by Powell, and adapted to the same eye-piece as the excellent object-glass by Ross. They both perform admirably, and the additional power gained by the former one is not only of considerable advantage, but it affords an opportunity of instituting comparative trials, so as to diminish the chances of error. Both these glasses will therefore be employed in the succeeding observations, and I shall avail myself of opportunities of testing the measurements previously given, and of recording the results when they seem to be of any consequence. The magnifying power of Powell's glass with the micrometer eye-piece is as nearly as possible nine hundred and eighty diameters, and the object is very distinctly defined.

After repeating very carefully numerous observations on the corpuscles in their own serum, as compared with specimens dried in the manner formerly described, it appears that the latter are almost always a little larger and more accurately defined in the outline than the former. This is particularly observable at the margins of the dried preparations, where the corpuscles are very thinly spread, and where desiccation takes place instantaneously when they are applied warm from the wound to the glass. Towards the centre, as the particles are more thickly aggregated, they do not dry so quickly, but have time to contract a little, and accordingly correspond in diameter pretty accurately with those observed in their own serum. I have noted instances in which they manifestly shrunk while under examination in the serum, as if they were acted on by the glasses between which they were placed, the edges of the disks becoming more rounded, occasionally granulated, and not unfrequently puckered or swollen, so that the central concavity in many of them was very remarkable, and often more or less misshapen from the bulging of the edges towards the centre, a triangular depression with concavity of the margins being thus occasionally produced on the surface of the corpuscles.

Though saline solutions are useful in diluting the blond for comparative observations, measurements from corpuscles so preserved arenot worthy of much reliance. The shrinking,

* Communicated by the Author. † L. \& E. Phil. Mag. for January 1840. 
or the alteration of form in the disks, may generally be observed even in the course of a minute or two after the mixture. Nor is the serum of one animal always proper to dilute the blood of another; for I could seldom get the corpuscles of the carnivora, or even of some ruminants, to mix well with the serum of the horse.

If obtained from the body a day or two after death, the disks are generally so clustered together as to be seen very indefinitely in the wet state, although some of the smallest detached from the masses are often tolerably distinct. 'The corpuscles are mostly very irregular in size, approaching more to the spherical shape, and even more susceptible of alteration from any of the common methods of dilution, than in blood procured from the living animal. By drying, however, a tolerably clear outline of the disks from the carcass may in most instances be procured, although every method sometimes fails, as I experienced a short time since in some blood from the Sloth Bear and from the Malay Sun Bear. Though the bodies of these animals were perfectly fresh, and the masses of fibrine in the heart and great vessels firmly coagulated, the particles of the blood were so much conglomerated, and their size so singularly variable, that it was impossible either by drying or any method of dilution to obtain even an approximation to their average diameter; and yet some corpuscles procured from the living Sloth Bear did not exhibit such irregularities.

Of the accidental circumstances by which the particles are liable to become enlarged, besides incipient putrefaction, the moisture of the atmosphere, of the breath, or of the hand are the most frequent. Dried or drying specimens are thus instantly injured or destroyed, the disks being more or less altered in shape and deprived of their colouring matter. But much depends on the degree in which these causes may have acted; for a diminution in the magnitude of the corpuscles may be the consequence. If, for example, some water be mixed with blood, the disks immediately become much enlarged and spherical, quickly losing their colouring matter; and yet if the whole of this be thus removed, after a while the outlines of the disks, very faint indeed, may frequently be recognised, diminished considerably in diameter and apparently quite flat. They may always be clearly seen by treating them with a strong solution of corrosive sublimate. The human blood corpuscles, thus enlarged at first, and then deprived of their colouring matter, and reduced in size, generally present a diameter of about 1-4800th of an inch, whether detected in the pure water or rendered more apparent by the sublimate. 
They have a very characteristic appearance, being remarkably flat and pellucid, several generally touching at their edges so as to form groups, scarcely ever turning over or even moving in the fluid. It is obvious from the size, shape, and general appearance of these particles, that they are not identical with those which have been usually described as the nuclei of the blood corpuscles. The average diameter of the disks in the first instance was 1-3429th of an inch.

Besides the precaution concerning the preservation of the blood, it is equally necessary to be careful as to how it is obtained. The corpuscles are more or less modified very quickly after extravasation; and if some delay occur, and the drop of blood, effiused into the subcutaneous tissue, require pressure to determine it to the surface, the corpuscles in that blood may be expected to be irregular in form and magnitude, muny of them particularly presenting a granulated appearance. Hence the glass should not be pressed upon the wound, but merely lighily touched on a drop of the blood, however small, that has appeared freely immediately after the puncture. A specimen thus procured, and dried as before noticed, will be excellent, although in all cases where it is practicable a small incision directly into a superficial vein will be preferable.

'The granulated particles are almost uniformly smaller than the common disks, and it is not inprobable that some of the former may be produced by the irregular shrinking of the latter. In some instances I could not detect any of the granulated corpuscles in the blood immediately after it was taken from the animal, although they were to be seen abundantly after a few hours' exposure in the serum to the atmosphere, the temperature ranging between $45^{\circ}$ and $50^{\circ}$. In one observation some of the extremely minute spherules, which are not uncommon in the blood, were observed to attach themselves to a few of the smaller disks, so as to produce the granulated appearance.

The blood corpuscles therefore are so singularly susceptible of variation in size and form from the operation of very slight agency, that there is probably no other microscopic object of equal delicacy, or that requires so much experience in its management. Hence it is not surprising that cursory observers should have committed remarkable errors, and that the history of the blood corpuscles, even after the labours of more careful inquirers, should have been so much obscured by discrepancies, particularly when it is considered how the inherent difficulties of the subject have been increased by the imperfection of instruments. Hewson indeed, whose obser- 


\section{$108 \mathrm{Mr}$. Gulliver's Observations on the Blood Corpuscles}

vations apart from his hypotheses are generally remarkable for their accuracy, laboured under these disadvantages, but there is reason to believe that his results were not obtained without the most devoted and diligent inquiry.

The discrepancies just alluded to are frequently adduced as instances of how little credit should be attached to microscopical anatomy generally. There does not appenr to me to be any reason in the objection; first, because it is seldom if ever urged by those who are sufficiently acquainted with the instrument to enable them to judge fairly of its use ; and secondly, the same objection might in like manner be made to the use of the unassisted vision in minute anatomy. Whoever has attended to the history of the elementary structure of different parts in the animal œconomy, as given by anatomists who have not employed glasses, must be acquainted with differences in the observations, just as remarkable as those which have resulted from the use of the microscope. But no one ever yet ventured to suggest that the imperfection of our senses was a reason why they should be dispensed with. The intimate structure of the bones, of the cellular or adipose tissues, are, among others, singularly obscured by false observations, notable for their number as well as for their disagreement. Yet the microscope has made these things infinitely more simple. No anatomist would now require to found the distinctions between the cellular and adipose tissues chiefly on remote physiological phænomena; no one would doubt the difference who had once seen the vesicles of the latter by the aid of the microscope. Haller could thus have immediately seen and shown to others the proof of that great discovery, which required so much labour to demonstrate. A curious collection might be given of the errors of anatomical observations made by the unaided vision as compared with those which have arisen from the use of the microscope*. But this subject is foreign to my purpose, and I have only to repeat the conviction which I have elsewhere expressed $t$, that the minute anatomy of the fluids, both healthy and diseased, is of the utmost importance, and that the steady and successful pursuit of this object will ultimately be the foundation of a new æra both in physiology and pathology.

76. Orang-outang, (Pithecus Satyrus, a female, about a third grown. All the following sizes very common: 1-3552nd,

* On the limits of vision with the best instruments there is an ingenious paper by Ehrenberg, of which we are indebted to Mr. Francis for an excellent English version. See Taylor's Scientific Memoirs, Part iv.

+ On the Softening of Fibrine, Med. Chir. Trans., vol, xxii. 
1-3429th, 1-3368th, 1-3309th, and 1-3200th. Extreme diameters $1-4000$ th and $1-3000$ th, though a very few considerably smaller might be seen in the serum. Blood from a vein of the fore arm.

77. Hoolock Gibbon, (Hylobates scyrites,) nearly fullgrown female. Most frequent size of the corpuscles 1-3200th, but very variable from 1-4570th to 1-2782nd of an inch. Blood from the left ventricle, dried, three days after death.

78. White Whiskered Gibbon, (Hylobates ? ?) a male, about two-thirds grown. Most frequent diameters of corpuscles 1-34,28th and 1-3200th. Extreme sizes 1-4570th and 1-2900th. In serum 1-4000th common; in weak saline solutions still smaller, and many of the edges swelled and punctured, so as to form a very remarkable triangular or quadrangular depression in the centre of the disks. Blood from the left ventricle of the heart.

I am informed by Mr. Ogilby that this is a new species of Hylobates.

79. Magot or Barbary Ape, (Papio sylvanus), 1-3428th and 1-3200th most frequently. Extreme sizes 1-4570th and 1-2900th. Blood from the aorta and vena cava.

80. Rhesus Monkey, (Macacus rhesus,) a young male. Corpuscles very variable in size, $1-3200$ th very common; extreme diameters 1-4000th and 1-2666th of an inch. Blood from left ventricle of the heart.

81. Toque or Chinese Bonnet Monkey, (Macacus radiatus), a male, about two-thirds grown. 1-3600th and 1-3200th common size of the corpuscles. Blood from the left ventricle.

82. Black Ape, (Macacus niger,) full-grown male. Most common diameter of the disks 1-3554th of an inch; extreme sizes 1-4572nd and 1-2965th. Blood from right ventricle.

83. Hare-lipped Monkey, (Macacus cynomolgus,) fullgrown male. Most common diameter of the disks 1-3429th of an inch; extreme sizes 1-4500th and 1-2666th. Blood from the renal vein.

84. Pigtailed Monkey, (Papio nemestrinus,) a female twothirds grown. Most common diameter of disks 1-3329th and 1-3555th. Extreme sizes 1-4570th and 1-2900th of an inch. Blood from the portal vein, from the coronary veins, and from the right ventricle.

85. Jacchus Monkey, (Jacchus vulgaris, ) adult male. Four measurements of three or four in a row, gave the following diameters of each disk : 1-3552nd, 1-3554th, 1-3693rd, and 1-3555th. Extreme diameters 1-4570th and 1-3000th. Blood from a prick of the tail. 


\section{$110 \mathrm{Mr}$. Gulliver's Observations on the Blood Corpuscles}

In the venous blood of the monkey tribe, besides lymph globules of the cominon size and appearance, there are generally spherical bodies of a very white colour, and frequently of semi-fluid consistence, as may be inferred from their being seen occasionally to alter in shape, like a drop of any viscid matter subjected to currents of particles on its margins. The white round bodies vary in diameter from 1.4000 th to 1-1777th of an inch. Their number is often very great, and they may be observed very remarkably in blood from the mesenteric veins. In the blood of the right ventricle they are also extremely common, though the semi-fluid appearance is more frequently seen in the former. These observations were made on monkeys, apes, and baboons, dead of various diseases, chiefly tubercular phthisis. Tubercle seems in these animals to be as common in the spleen as in the lungs; indeed, this deposit is not unfrequent in the former organ when none can be found in the latter.

86. European Brown Bear, (Ursus Arctos, ) female, hardly a third grown. Most frequent diameters of corpuscles 1-3600th, 1-3692nd, 1-3750th, 1-3790th, and 1-4000th. Extreme sizes 1-4570th and 1-3048th. Blood from a prick of the upper lip.

87. Black Bear, (Ursus Americanus, a male, nearly same size and apparently of the same age as the European Bear. All the following diameters common: 1-3600th, 1-3693rd, 1-3790th, 1-3840th. Extreme sizes 1-4570th and 1-3000th of an inch. Blood from a prick of the upper lip.

88. Cinnamon or Chocolate Bear, (Ursus Americanus, var.?) a male, from North America, apparently of the same age as the Black and European Bears. 1-3693rd, 1-3790th, 1-3840th, 1-4000th, all common sizes. Extreme diameters 1-4800th and 1-3000th. Blood from a prick of the upper lip.

89. Polar Bear, (Ursus Maritimus, an old female. The following sizes very common : 1-3600th, 1-3693rd, 1-3764th, and 1-3840th. Extreme diameters 1-4570th and 1-3048th. There were seen, though rarely, some as small as 1-5333rd of an inch. Blood from a prick of the upper lip.

90. Sloth Bear (Ursus labiatus, an aged female and a fullgrown male. Most common diameter of corpuscles from 1-4000th to 1-3555th. Extreme sizes 1-4800th and 1-3000th of an inch. Blood from the different cavities of the heart of the female; from a prick of the lip of the male, which was a healthy animal. The unsatisfactory result of the examination of the corpuscles in the female has been already noticed; the specimen from the male was not a very good one.

91. Raccoon, (Procyon lotor, ) nearly full-grown male. In the dried corpuscles, the following sizes all very common: 
1-4500th, 1-4.572nd, and 1.4800th. Extreme diameters, $1-6000$ th and 1-4,000th. In the serum, a great many of the disks 1-6000th to 1-5333rd of an inch. Blood from a vein of the fore foot.

92. Wolf, (Canis Lupus, ) adult male. 1.-3554th, $1=3635$ th, 1-3692nd, all very frequent diameters. Extreme sizes 1-4570th and 1-3000th. Blood from a prick of the ear.

93. Jackal, (Canis mesomelas, ) adult female. The following diameters very common: 1-3552nd, 1-3600th, 1-3693rd, 1-3790th. Extreme sizes 1-4570th, 1-3000th. Blood from a prick of the ear.

94. Two Spotted Paradoxure, (Paradoxurus binotatus,) a female nearly full-grown, from Western Africa. The following sizes frequent: 1-4572nd, 1-4800th, and 1-5052nd of an inch. Extreme diameters 1-6000th and 1-3555th. Blood from a prick of the tail.

95. Striped Hyæna, (Hycena vulgaris,) female not quite full-grown. 1-4000th, 1-3764th and 1-3552nd, common diameters. Extreme sizes 1-4800th and 1-3000th. Blood from a vein of the ear.

96. Lion, (Felis Leo,) from Africa, nearly full-grown. The most common diameters 1-4500th and 1-4365th of an inch. Extreme sizes 1-5800th and 1-3554th. Blood from a prick of the ear.

a. Lioness, about two-thirds grown. Some corpuscles obtained from the cutaneous vessels of the leg gave the same measurements.

97. Puma or Silver Lion, (Felis concolor, ) from South America, full-grown male, 1-4572nd, 1-4500th and 1-4440th, the most frequent diameter's of the disks. Extreme sizes 1-5800th and 1-3554th. Blood from a prick of the ear.

98. Tiger, (Felis Tigris, from India, a female, full-grown. Common sizes 1-4440th, 1-4.210th, and 1-4268th. Several also of 1-4000th. Extreme diameters 1-5333rd and 1-3428th of an inch. Blood from a vein of the ear.

The size therefore of the blood corpuscles of these larger species of the genus Felis is very nearly alike*. In some of the smaller species, as the Cat (27.), Serval (28.), and Lynx (30.), the disks have much the same diameter, as I infer from frequent examinations. Mr. Siddall too, before he was acquainted with the result of my observations, came to the same conclusion, from several trials with the blood of the cat, as compared with one specimen of that of the tiger. In some blood obtained during life from the femoral vein and from the femoral artery of a cat, about a third-grown, the disks most 
commonly presented the following diameters, 1-4.365th and $1-4752$ d d of an inch; and there were several 1-4000th, the thickness of the edges of the disks being 1-16,000th. The blood was examined quickly after it was obtained, and no appreciable difference was seen between the arterial and venous corpuscles. They are certainly but very slightly smaller than in the tiger.

99. A Kangaroo, (Macropus Eugenii?) a female, 1-3554th, 1-3432nd, and 1-3200th, a common diameter of the disks, and 1-4000th and 1-3000th rather frequent sizes. The edges of the corpuscles generally from 1-12,000th to 1-10,000th of an inch thick. Blood from a prick of the tail.

100. The Coypu, (Myopotamus Coypus,) an adult. Most common diameters of corpuscles 1-3500th and 1-3200th of an inch. Small disks, 1-4000th; large, 1-2666th, in the dry state. Thickness of the edges of the corpuscles 1-12000th to 1-9600th. In the serum, the corpuscles were more variable in size, 1-4572nd and 1-3000th of an inch being very frequent. Blood from a prick near the buttocks.

101. Gray Squirrel, (Sciurus cinereus, ) adult male. Common diameters 1-4266th, 1-4000th, 1-3840th, and 1-3600th. Extreme sizes 1-6000th and 1-3000th. Magnitude of corpuscles very irregular; a few not more than 1-6400th. Bloor from a prick of the nose for the first examination, and from the ear a few days subsequently for another trial.

102. Capistrated Squirrel, (Sciurus capistratus, ) adult male. Common diameters 1-4000th, 1-3790th, and 1-3693rd. Extreme sizes 1-5333rd and 1-3000th. A few of the very small corpuscles less than 1-6400th of an inch in diameter. Blood from a prick of the upper lip.

103. Black Squirrel, (Sciurus niger,) an adult. All the following sizes frequent, 1-3600th, 1-3692nd, 1-3790th, and 1-3840th. Extreme diameters 1-5333rd and 1-3000th. A few of the very small corpuscles were seen.

The size of the corpuscles, as far as I have observed, is very irregular in the genus Sciurus. There are circular particles, though not in large numbers, yet very remarkable for their regularity and diminutive size in regard to the common blood disks. For this reason the very small corpuscles should be further examined. I have certainly seldom seen them so remarkably in any other genus, though I think they appeared in the blood of the female Sloth Bear. In the Palm Squirrel (72.) there were several about 1-7000th of an inch. 104. Wild Boar, (Sus Scrofa) from Asia, male, nearly full-grown. 1-4266th, 1-4365th, and 1-4000th, most frequent 
diameters of the disks. Extreme sizes 1-1533rd and 1-3555th, Blood from a prick of the nose.

105. Collared Peccari, (Dicotyles torquatus,) from Mexico. Full-grown female. 1-4173rd, 1-4500th, 1-4572nd, and 1-4800th very common diameters of disks. Extreme sizes 1-6000th and 1-3555th. Múch more irregular in size than in the Wild Boar, and from two examinations appearing to be smaller. Blood from a prick of the upper lip.

106. Zebra, (Equus Burchellii, ) full-grown female. Average-sized disks 1-4500th; and 1-4.800th, 1-4.365th and 1-4000th, not uncommon. Extreme sizes 1-5800th and 1-3368th. Blood from a prick of the nose.

107. Dshikketai or Wild Ass, (Equus Hemionus), a female, as large as a common Ass. The most frequent sizes of corpuscles 1-4572nd and 1-4800th. Several as large as 1-4000th. Extreme diameters 1-5800th and 1-3555th of an inch. Blood from a prick of the nose.

With reference to the blood of the Horse (see No. 34 in the former paper) Mr. Siddall obtained the following measurements of the common-sized corpuscles from a cart stallion, aged 12 , suddenly killed : 1-4.360th, 1-4.208th, and 1-4.362nd. The blood, from a vein of the testicle, examined soon after the death of the animal.

108. Axis Deer, (Cervus Axis, ) adult male. Most frequent sizes 1-4924th and 1-5333rd of an inch. Extreme diameters 1-6000th and 1-4365th. Altogether very irregular in size. Blood from a vein of the ear.

109. A Deer, (Cervus macrourus?). Most frequent diameters 1-5142nd and 1-5333rd. Extreme sizes 1-6400th and 1-4000th. An adult male and female; the blood from the renal vein of the former, and from the ear of the latter.

These animals were shipped at Honduras, and brought home with the Mexican Deer.

110. Reeves's Muntjac, (Cervus Reevesii, Ogilby). The corpuscles belonging to the same class as those of the Porcine and Mexican Deer. 'They will be all fully described together on a future occasion.

111. Sing Sing Antelope, (Antilope Sing Sing,) adult female. Corpuscles very variable from 1-6000th to 1-4.000th 0. an inch; 1-4800th was very common, and 1-5333rd common. Blood from a prick of the nose.

112. Nyl-ghau, (Antilope picta,) a young mare, hardly half-grown. 1-4.924th, 1-4800th and 1-4572nd, all common diameters. Extreme sizes 1-6000th and 1-4365th of an inch. Blood from a vein of the ear.

113. Cervine Antelope, (Antilope bubalis,) adult male. CorPhil. Mag. S. 3. Vol, 16. No, 101, Feb, 1840. 


\section{On the Blood Corpuscles of the Mammiferous Animals.}

puscles remarkably variable in size, and differing much whether examined dry or in their serum. In the former state 1-5333rd and 1-6000th most common diameters, and extreme sizes 1-6400th and 1-4562nd. In the serum 1-6856th the most frequent size. Blood obtained for the first examination from a prick of the nose, and for the second some weeks afterwards from an incision of the ear. The animal was diseased.

114. Buffalo, from Manilla, (Bos Bubalus, ) adult female, 1-5142nd, 1-4800th, and 1-4500th common diameters. Extreme sizes 1-5333rd and 1-3600th. Average thickness of the edges of the disks 1-14,000th of an inch. Blood from a vein of the ear.

115. Cape Buffalo, (Bos Caffre, ) full-grown male. 1-514.2nd and 1-4800th, most frequent sizes in the dried specimens. Extreme diameters 1-6000th and 1-3554th. The corpuscles in their serum were commonly 1-5333rd, or even as small as 1-600uth of an inch. Blood from a vein of the ear.

From two trials it appeared that disks were slightly smaller than in Bos Bubalus.

Napu Musk Deer (49.) An adult fenale having lately died at the Zoological Gardens, I availed myself of an opportunity of examining some blood from the different cavities of the heart, as well as from the cava, portal, and mesenteric veins. The result fully confirms the accuracy of my former observations*, especially that the blood corpuscles of the Musk Deer are smaller than any previously described in the mammalia. In relation to the parts from which the blood was obtained, there was no appreciable difference in the disks. The average diameter of those procured from the dead animal was 1-13,400th of an inch.

In an animal with blood corpuscles so remarkably minute, it was interesting to ascertain the comparative magnitude of the lymph globules. The latter were therefore carefully examined with this view; and their size, appearance, and chemical characters found to be identical with the lymph globules of many mammals with large blood particles. The lymph granules in the Napu Musk Deer varied in diameter from 1-5000th to 1-3500th of an inch. Hewson inferred from his observations that there was a relation between the particles of the lymph and blood in the same animal, and a difference in the size and shape of the former in different animals.

I have retently examined the blood of the Vicugna (37.), and compared it again with that of the Dromedary (36.). In

* Dublin Medical Press, Nov. 27, 1839. L. and E. Phil, Mag. Dec. 1839, and Jan. 1840. 
the former the following measurements of the corpuscles were taken. Long diameters, $1-4000$ th very common, many $1-3555$ th, and a few, not commonly seen, $1-2666$ th. Several considerably shorter than 1-4000th of an inch, for instance, 1-5333rd; and even 1-6000th very rarely. Short diameters most firequently 1-6400th and 1-7110th. The shorter corpuscles are generally broader in proportion than the others, some nearly circular, but in this observation none perfectly so. Mr. Siddall's measurements agree as nearly as possible with mine, and the corpuscles in the Vicugna appear to be a little smaller than those of the Dromedary. Though taken from a vein of the ear, the blood was of a bright brick-red colour, as it appeared from the puncture. It would be singular if the venous blood of any of the mammals with oval particles should not have the usual dark colour.

'The Rhinoceros (52, p. 32.) 1-2554th is a misprint for 1-3554th.

The Red American Fox (24) is the Canis fulvus, not a variety of C. Vulpes.

XIX. On Galvanic Series formed of Zinc and Inactive Iron. By $M r$. Thomas Hawkins.

To the Editors of the Philosophical Magazine and Journal. Gentlemen,

NE of Professor Schœenbein's experiments described at $p$. 429 of vol. x. of the L. \& E. Phil. Mag., by which he obtained an electric current of high intensity with wires "having one of their ends coated with peroxide of lead, and each end put into a separate vessel filled with nitric acid, a hundred times diluted," interested me so much, that I was led, at the time of its publication, to make some experiments, with the view of establishing the possibility of employing iron in the peculiar condition as the negative metal in voltaic batteries. After some failures I succeeded in forming a galvanic circle with a fine iron wire put into nitric acid of sp. gr. 1.5 contained in a tube of plaster of Paris, and associated with amalgamated zinc in diluted sulphuric acid, which electrolyzed water; and two of such circles, in series, liberated the gases rapidly, continuing in action for seven hours, when sudden effervescence of the acid occurred and the wires were dissolved. Efficient circles, but of less permanency, were constructed of iron wires alone, having one of their ends in the porous cells of nitric acid, the other in diluted sulphuric or even very diluted nitric acid. The liability of the iron to be suddenly attacked by the nitric acid was prevented, so far as my experience went, by dissolving mercury in the acid, in accordance with the Pro- 